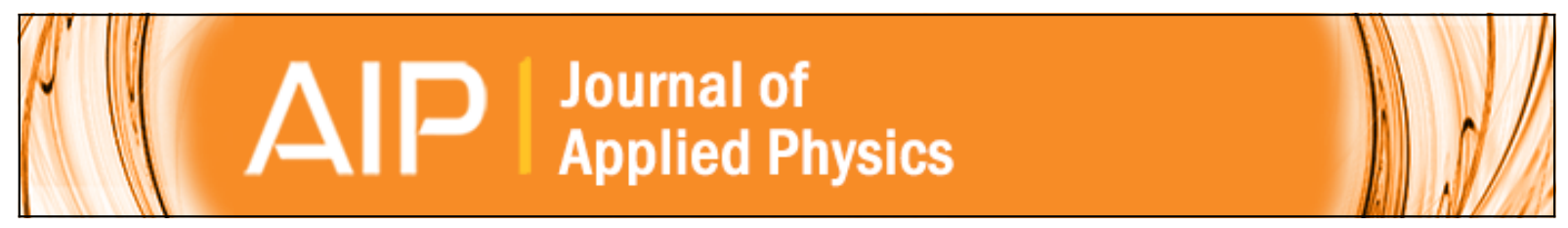

\title{
Wavelength-sized cavities in high aspect InP/InGaAsP/InP photonic crystals
}

H. H. J. E. Kicken, I. Barbu, R. W. van der Heijden, F. Karouta, R. Nötzel, E. van der Drift, and H. W. M.

Salemink

Citation: Journal of Applied Physics 106, 083106 (2009); doi: 10.1063/1.3246865

View online: http://dx.doi.org/10.1063/1.3246865

View Table of Contents: http://scitation.aip.org/content/aip/journal/jap/106/8?ver=pdfcov

Published by the AIP Publishing

\section{Articles you may be interested in}

Quasiresonant excitation of $\mathrm{InP} / \mathrm{lnGaP}$ quantum dots using second harmonic generated in a photonic crystal cavity

Appl. Phys. Lett. 101, 161116 (2012); 10.1063/1.4761248

Room temperature, continuous-wave coupled-cavity InAsP/InP photonic crystal laser with enhanced far-field emission directionality

Appl. Phys. Lett. 99, 091110 (2011); 10.1063/1.3633120

High quality GalnP nonlinear photonic crystals with minimized nonlinear absorption

Appl. Phys. Lett. 95, 221108 (2009); 10.1063/1.3269998

Fabrication of high-quality-factor photonic crystal microcavities in InAsP/InGaAsP membranes

J. Vac. Sci. Technol. B 22, 875 (2004); 10.1116/1.1701848

Threshold dependence on the spectral alignment between the quantum-well gain peak and the cavity resonance in InGaAsP photonic crystal lasers

Appl. Phys. Lett. 83, 4107 (2003); 10.1063/1.1627466

\section{A|P| $\left.\right|_{\text {Applied Physics }} ^{\text {Journal of }}$}

Journal of Applied Physics is pleased to announce André Anders as its new Editor-in-Chief 


\title{
Wavelength-sized cavities in high aspect InP/InGaAsP/InP photonic crystals
}

\author{
H. H. J. E. Kicken, ${ }^{1, a)}$ I. Barbu, ${ }^{1}$ R. W. van der Heijden, ${ }^{1}$ F. Karouta, ${ }^{1}$ R. Nötzel, ${ }^{1}$ \\ E. van der Drift, ${ }^{2}$ and H. W. M. Salemink ${ }^{2}$ \\ ${ }^{1}$ COBRA Research Institute and Center for NanoMaterials, Eindhoven University of Technology, \\ P.O. Box 513, NL-5600 MB Eindhoven, The Netherlands \\ ${ }^{2}$ Kavli Institute of Nanoscience, Delft University of Technology, P.O. Box 5053, NL-2600 GB Delft, \\ The Netherlands
}

(Received 24 July 2009; accepted 14 September 2009; published online 22 October 2009)

\begin{abstract}
The photonic properties of two classes of wavelength-sized cavities are reported for deeply etched $\mathrm{InP} / \mathrm{InGaAsP} / \mathrm{InP}$ planar photonic crystals. The high aspect, deeply etched structures are studied as potential building blocks for nonmembrane type photonic devices in standard InP photonic integrated circuits. The first class consists of cavities of one unit cell in one direction and varying size in the other planar direction. The studied class includes a Fabry-Perot type cavity with one row of missing holes, a simple single missing hole defect cavity, and a cavity consisting of two holes which have been slightly shifted and reduced in hole radius. The best observed quality factor of 65 in this class is obtained for a single hole defect cavity. The second class is comprised of cavities which are derived from a three missing row defect in one direction and varying size in the other direction. This includes a Fabry-Perot type cavity with three rows of missing holes, a point defect cavity consisting of seven unetched holes and a six hole ring cavity. The best observed quality factor of 300 is obtained for the ring cavity in this second class of structures, which is adequate for applications. (C) 2009 American Institute of Physics. [doi:10.1063/1.3246865]
\end{abstract}

\section{INTRODUCTION}

Recently, photonic crystals (PCs), a periodic arrangement of dielectrics, attract a lot of attention due to their extraordinary optical properties. ${ }^{1}$ A practical implementation of PCs is a lattice of air cylinders etched into a semiconductor heterostructure slab waveguide (WG), so-called planar or two-dimensional (2D) PCs. The confinement to the plane is realized by the refractive index contrast based on total internal reflection. The InP/InGaAsP/InP system ${ }^{2}$ is a system of great practical importance for photonic integrated circuits (PIC) operating at telecom wavelengths $(1.55 \mu \mathrm{m})$.

Due to the low refractive index contrast, the guided mode has a large extension $(\sim 500 \mathrm{~nm})$ into the cladding layers, which requires the fabrication of deep holes to completely intersect the electromagnetic field. The air holes have to be etched well through the complete layer stack, to a depth of at least $2 \mu \mathrm{m}$, which is technologically challenging as it requires aspect ratios in excess of 12 . The required etch depth is typically much larger than required for membranes. However, compared to membranes, these deeply etched structures are mechanically robust, can be fabricated into standard photonic integrated circuitry, and active components may dissipate heat easily.

Deeply etched structures in the InP system are intrinsically lossy since the band gap is practically entirely located above the lightline for the perforated cladding, even for infinitely deep holes and infinitely thick cladding on both sides of the core layer. This makes these high aspect ratio components less suitable for applications which require cavities

${ }^{a)}$ Electronic mail: h.h.j.e.kicken@tue.nl. with ultrahigh quality $(Q)$ factors. The small size of the envisioned PC building blocks in conventional PIC limits the overall loss.

Resonant cavities are of particular importance for PIC applications, where they find diverse applications such as components for laser and sensors. Even for moderate $Q$ factors, cavities have applications such as add/drop filters, minimizing waveguide bending loss and broadband coupling. ${ }^{3-5}$ In particular a high efficiency broadband coupler could be achieved with a $Q$-factor of $\sim 160{ }^{3}$ The smallest size cavities in dielectrics are of the order of the cube of the wavelength, which is approximately the size of a unit cell in a PC.

The smallest defect cavities in PCs therefore consist of a single unetched hole (H1) or of one or two modified holes (H0). In the deeply etched system, the smallest cavities reported so far are line defects consisting of a single row of unetched holes, ${ }^{2,6,7}$ i.e., a Fabry-Perot (FP)-like cavity, and PC waveguides terminated by PC mirrors. ${ }^{8}$ Smaller defect cavities have so far only been reported for membranes in case of the $\mathrm{H} 0$ cavity $^{9}$ and in the case of the $\mathrm{H} 1$ cavity for both membrane ${ }^{10}$ and half membrane structures. ${ }^{11}$ Since both the $\mathrm{H} 0$ and the $\mathrm{H} 1$ cavity, as well as the single missing row FP cavity (FP1), occupy a single row of holes in one direction, we classify them as a single row defect class of cavities.

If the radius of the $\mathrm{H} 1$ cavity is extended to the neighboring unit cells, the slightly larger $\mathrm{H} 2$ cavity (seven unetched holes) is obtained. This cavity and the smallest ring cavity, formed by addition of a center hole into the $\mathrm{H} 2$ cavity, extends over three rows of the PC. In the $\Gamma M$ direction, as indicated in Fig. 1, these cavities are of equal size as the FP3 cavity (three unetched rows). This class of cavities is expected to have a higher $Q$ factor due to its larger size. So far, $\mathrm{H} 2$ cavities were reported in several membrane systems 


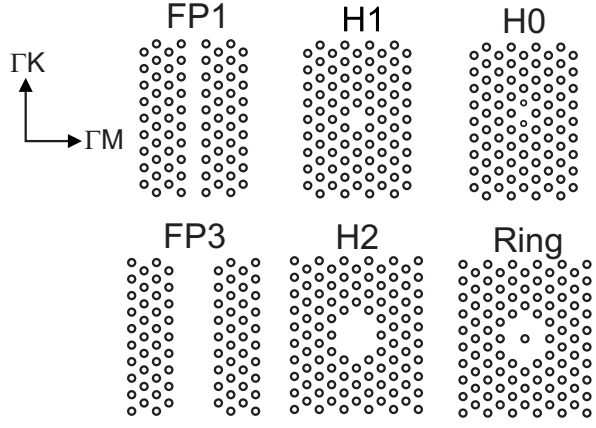

FIG. 1. Overview of the different cavity geometries. The top row shows the single row defect cavities, form left to right: FP1, H1, and $\mathrm{H} 0$ cavities. On the bottom the three row defects from left to right: FP3, H2, and ring cavities. The cavities are probed by measuring transmission in the $\Gamma M$ direction via mode access waveguides.

[InP (Refs. 10 and 12) and GaAs (Ref. 13)] and also for AlGaAs/GaAs half-membranes. ${ }^{11}$ The ring cavity is reported in Si (Refs. 14 and 15) and InGaAsP (Ref. 16) membranes.

A brief report of our work on $\mathrm{HO}$ and $\mathrm{H} 1$ cavities is given elsewhere. ${ }^{17}$ In the present work we investigate the properties of both cavity classes in the technologically important deeply etched InP system. The gradual change in the FP-like cavities (FP1 and FP3) by stepwise reduction in the transverse dimension is experimentally investigated by transmission spectroscopy and analyzed using finite difference time domain (FDTD) calculations. In addition, for the large cavities, the reflection of the PC is studied by analysis of FP fringes of both PC cavities and ridge WG (RWG) cavities terminated by a PC mirror. Also, the dependence of the $Q$-factor on thickness of the PC mirror was studied for the H2 cavity.

\section{EXPERIMENTAL}

In Fig. 1 a sketch of the different cavities, which were investigated experimentally, is given. The top row shows the class of cavities designated as "single row defect" cavities: FP1, H1, and H0; the bottom row displays the class of cavities designated as the "three row defect" cavities: FP3, H2, and ring cavities. The cavities of each row can be transformed into its neighbor on the right by addition of holes.

The PCs were fabricated by etching a hexagonal lattice of holes into a double heterostructure WG slab, consisting of $0.5 \mu \mathrm{m}$ InP upper cladding, $0.5 \mu \mathrm{m}$ InGaAsP guiding layer, and $1 \mu \mathrm{m}$ buffer layer as lower cladding on a InP substrate, using inductively coupled plasma reactive ion etching with $\mathrm{Cl}_{2} / \mathrm{O}_{2}$ chemistry. ${ }^{18}$ These structures were placed between 2.5- $\mu \mathrm{m}$-wide access RWGs to allow for transmission spectroscopy [see Fig. 2(a)]. The lattice constant was varied from 256 to $481 \mathrm{~nm}$ (lithographic tuning), and the designed air filling factor was chosen to be 0.3 . The hole depth for holes of $200 \mathrm{~nm}$ diameter is approximately $2.5 \mu \mathrm{m}$, an aspect ratio of $\sim 12$ [see Fig. 2(b)]. The scanning electron microscopy (SEM) picture displays a cross section of a typical device used for transmission spectroscopy. Transmission spectroscopy was carried out in an end-fire technique using a tunable laser in the range of 1470-1570 $\mathrm{nm}$ in transverse electric

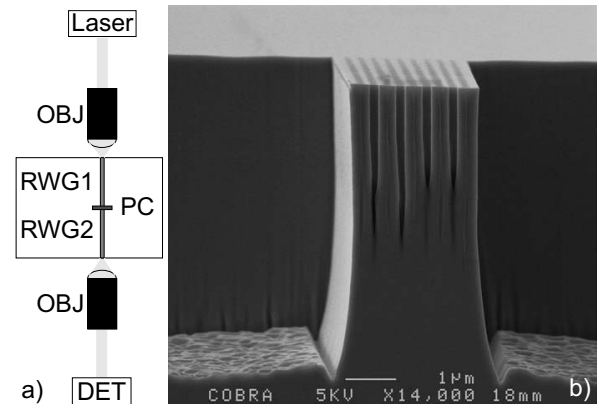

FIG. 2. (a) Schematic representation of the experimental setup. Laser light, tunable from 1470 to $1570 \mathrm{~nm}$ is focused by an objective (OBJ) on the cleaved facet of the RWG1 and guided toward the PC. The light exiting the RWG2 on the other side is collected by a second objective and detected (DET). (b) SEM picture of the cross section of the holes in a PC. The structure shown has a lattice constant of $337 \mathrm{~nm}$. The holes have a diameter of $200 \mathrm{~nm}$ and a depth of $\sim 2.5 \mu \mathrm{m}$. In the background the edges of the etched trenches, which are used to define the access WGs are visible.

(TE) polarization. The cavities depicted in Fig. 1 are probed by transmission in $\Gamma M$ direction, i.e., from left to right.

\section{RESULTS AND DISCUSSION}

\section{A. Single row defects}

In this section the single row defects are systematically investigated, starting from an FP1 cavity, as detailed in Fig. 1. This cavity is sketched in Fig. 3(a) by the uncolored holes. To obtain the H1 cavity, the FP1 cavity is modified by adding holes from both sides, leaving only the center hole unetched. For each of the set of the colored holes, the radii $r^{(4)}, r^{(3)}$, and $r^{(2)}$ are successively and gradually increased from 0 to $r$, the radius of the bulk crystal (white holes), i.e., first $r^{(4)}$ is increased from 0 to $r$, next $r^{(3)}$, etc. The holes with radius $r^{(4)}$ extend to the edge of the PC. Finally, the resonance frequency of the H0 cavity is investigated by modification of the colored holes in Fig. 3(b) with radius $r^{\prime}$ and shift $s^{\prime}$. The impact of the addition the holes and the gradual increase in the radius on the resonant frequency is studied by 2D-FDTD calculations. For PCs in low refractive index contrast systems, the $Q$-factor, which is equal to $\lambda / \Delta \lambda$, of the resonance peaks are limited to a few hundred and resonant frequencies are well reproduced by 2D-FDTD calculations with effective index approximation. ${ }^{19}$

Figure 3(c) shows the behavior of resonant modes of a PC system containing a single row defect as obtained from 2D-FDTD calculations (black lines). The lines connect the resonance frequencies found by 2D-FDTD calculations of the transmission of the PC and RWGs; each line consists of eight calculations per panel. Due to the excitation with RWGs, modes with a large coupling to the waveguide mode are preferentially excited.

From left to right in Fig. 3(c) (normalized frequency versus hole radii), the defect cavity is transformed from a FP cavity with one missing row of holes (FP1) to an H1 cavity by successive addition of holes with radii $r^{(4)}, r^{(3)}$, and $r^{(2)}$, as sketched in Fig. 3(a). In the right-most panel of Fig. 3(c) the resonance frequency of the $\mathrm{H} 0$ cavity is shown as a function of the radius $r^{\prime}$ of the two holes with radius $r^{\prime}$ and shift $s^{\prime}$ designated in Fig. 3(b). At selected positions, indicated by 

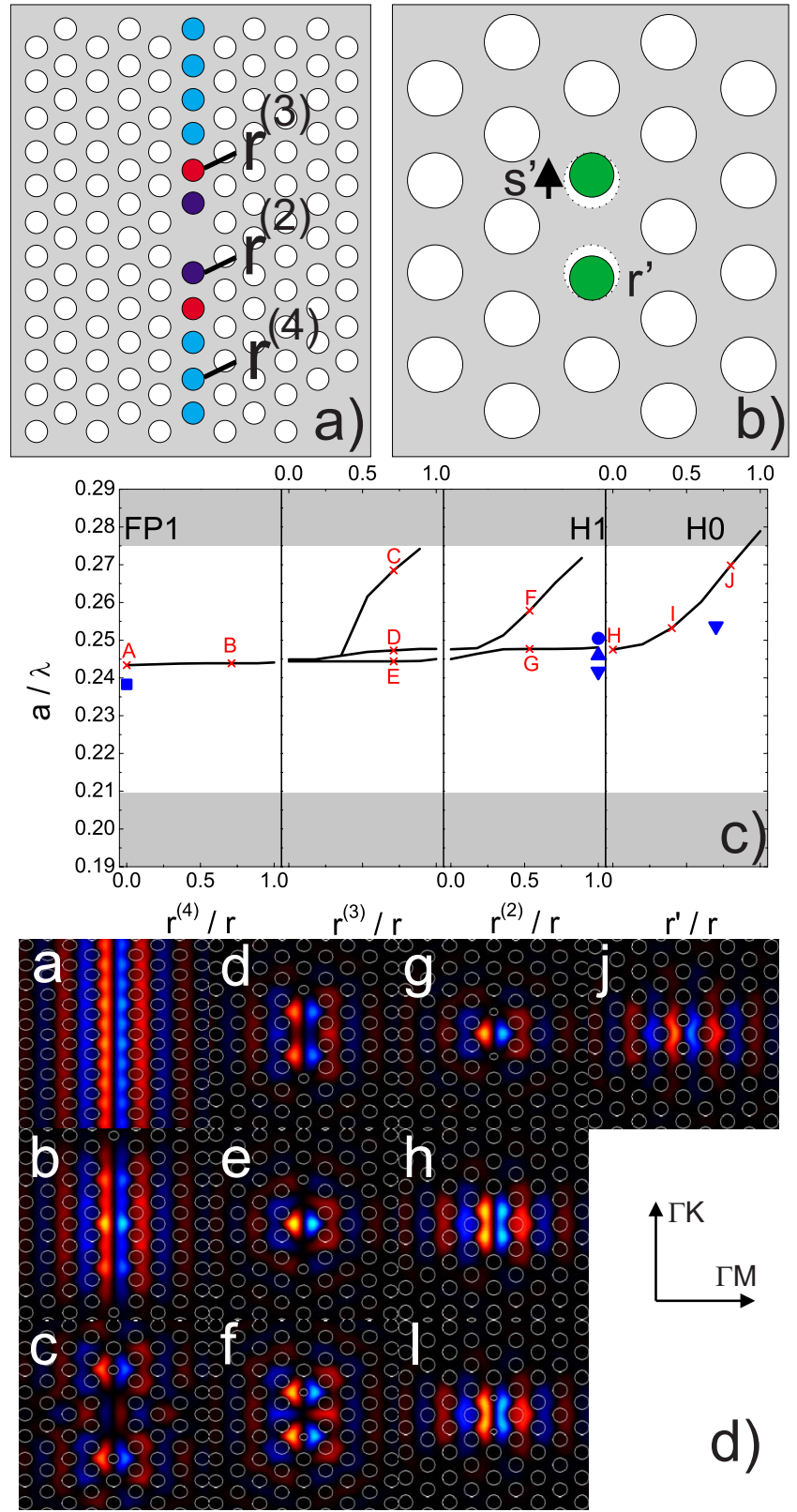

FIG. 3. (Color online) (a) By addition of the colored holes with radii $r^{(4)}$, $r^{(3)}$, and $r^{(2)}$ respectively a FP1 cavity is transformed into an H1 cavity. (b) Detail of the formation of an $\mathrm{H} 0$ cavity. The colored holes are shifted by $s^{\prime}$ which is related to the radius $r^{\prime}$ by $s^{\prime}+r^{\prime}=r$, with $r$ the radius of the holes of the bulk PC. (c) The evolution of the resonant modes of a single row defect cavity. The FP1 cavity is transformed into a $\mathrm{H} 1$ cavity by addition of PC holes as depicted in (a), ultimately a H0 cavity is formed. The horizontal axis gives the ratio of the hole radii, $r^{(4)}, r^{(3)}, r^{(2)}$, and $r^{\prime}$ with respect to the bulk hole radius $r$; the tick labels are displayed below and above alternating. The squares, triangles, and circles indicate the available experimental data, with each different symbol representing data from a different fabrication cycle. The vertical axis gives the normalized frequency. The gray areas denote the dielectric and air band. (d) Magnetic field maps $\left(H_{y}\right)$ of the various modes indicated by the crosses and capital letters in $\mathrm{b}$. The mode patterns $\mathrm{a}-\mathrm{m}$ correspond to $\mathrm{A}-\mathrm{M}$ indicated in (c).

the crosses and capitals, the modal field pattern $\left(H_{y}\right.$, magnetic field, which is perpendicular to the plane for TE modes) is displayed in Fig. 3(d); the letters a-j correspond to A-J in Fig. 3(c). The gray shaded areas indicate the transmission bands. The experimental peak positions obtained by transmission measurements from several samples, are shown in

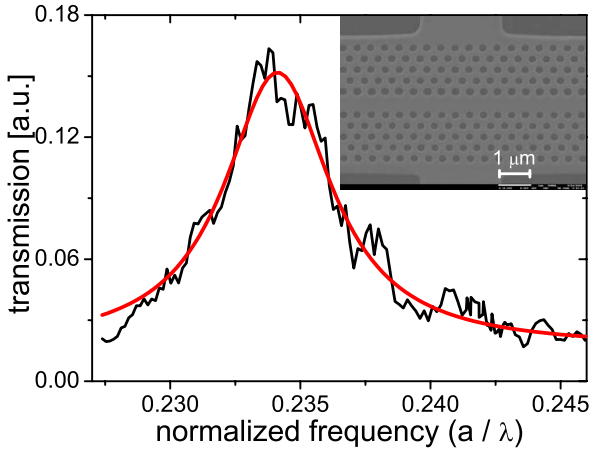

FIG. 4. (Color online) Spectrum of a FP-type defect cavity with one missing row. The bold line is a Lorentzian fit used to accurately determine the $Q$-factor, which is found to be 47 . The inset displays a SEM picture of the structure.

squares, circles, and triangles. The spread of the resonance frequency from sample to sample is due to different fabrication runs. The results from these measurements will be discussed in further detail below.

The FP1 cavity $\left[r^{(4)}=0\right]$ has a single resonant mode roughly in the center of the band gap, with a modal field pattern [see mode a in Fig. 3(d)], characteristic for a FP mode. The experimental spectrum of the resonance is displayed in Fig. 4, with a SEM picture of the device as an inset. Fitting of the resonance peak (bold line) yields a $Q$-factor of 47 . This $Q$-factor compares favorably to previously reported results of FP-type cavities of similar width, where $Q$-factors of 20-30 were found. ${ }^{2}$ More recently, optimization in etching (chemically assisted ion beam etching) has led to an increase in the $Q$-factor for the FP1 to approximately $300,{ }^{6,7}$ which is primarily attributed to increased hole depths. The measured resonance frequency is slightly lower than obtained by the 2D-FDTD calculations, which is due to a difference in designed $r / a$ value.

By steadily increasing radius $r^{(4)}$ the resonant mode is compressed in the direction parallel to the defect row, showing a pattern with a higher field concentration in the center of the PC [see mode b in Fig. 3(d)]. The resonance frequency and the modal pattern remain essentially unchanged. As the next set of holes is introduced and their radii $r^{(3)}$ are successively increased, the mode splits into three separate dipolelike modes which have distinct modal patterns [c, d, and e in Fig. 3(d)]. The lowest frequency mode e is the zeroth order mode with a high field concentration in the center of the PC. Both higher order modes have extra nodes along the $\Gamma K$ direction. The second order mode $\mathrm{c}$ is concentrated on the $r^{(3)}$ holes, which explains the strong dependence of the resonance frequency on the radius. This mode disappears from the band gap when $r^{(3)}$ approaches $r$.

As the modes are more confined by the increase in $r^{(3)}$ and subsequently $r^{(2)}$, the splitting of the two lowest order modes is increased after the next set of holes is added and their radii $r^{(2)}$ increased. Since the first order mode is concentrated near the $r^{(2)}$ (blue) holes, it is driven toward the air band. At $r^{(2)} / r=1$ the $\mathrm{H} 1$ cavity is obtained, which has only one (degenerate) mode. ${ }^{20}$

The experimental spectrum of an $\mathrm{H} 1$ defect cavity is shown in Fig. 5. The frequencies of three such cavities, ob- 


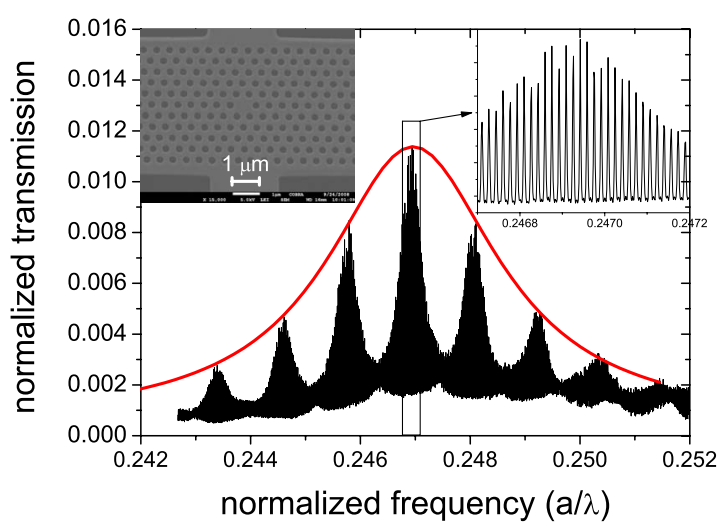

FIG. 5. (Color online) Spectrum of an $\mathrm{H} 1$ defect cavity. The bold line is a Lorentzian fit of the envelope, used to obtain a $Q$-factor of 65 . In the inset a SEM micrograph of the H1 structure is shown. The modulation, beat and fine fringes, which are detailed in the inset, are caused by reflections at the cleaved facets of the RWGs and the PC in between and are discussed in section $\mathrm{B}$.

tained from independent fabrication runs, is plotted in Fig. 3(c). The resonance feature is modulated by a large beat and fine FP fringes originating from the cavities formed by the cleaved facets and the PC in between ${ }^{21}$ (resolved in the right inset). The frequency of the fine fringes is due to the length of the RWGs and the frequency of the beat is caused by the length difference of the RWGs. From a Lorentzian fit of the envelope (bold line) the $Q$-factor of the $\mathrm{H} 1$ cavity is determined to be 65 . In these case no antireflection coating was applied. the function of the antireflection coating is to suppress FP modulations arising from reflections at the samples edges; As a result of this coating, the signal approaches the envelope, as in Fig. 4.

As seen from the evolution of the mode patterns from point A via B, E, and G [modes a, b, e, and g in Fig. 3(d)], the character of the modes does not significantly change, i.e., the modes have a node in the center of the cavity and no node in the $\Gamma K$ direction. The main difference among the modes is the gradual confinement of the field into the center of the cavity. For all these modes the primary confinement direction is in the $\Gamma M$ direction. Therefore it is reasonable to analyze the $\mathrm{H} 1$ as a FP1 cavity. The $\mathrm{H} 1$ cavity is confined in two dimensions while the FP cavity is only confined in one dimension, therefore the FP cavity is expected to yield lower losses. From the FP analysis using the $Q$-factor a value for the reflectivity of the $\mathrm{PC}$ mirror can then be determined. This will yield a lower bound on the reflectivity of the PC mirror of the $\mathrm{H} 1$ cavity. The reflectivity can be calculated from

$$
Q=m \pi \frac{\sqrt{R}}{1-R}
$$

with $m$ the mode number associated with the cavity length and $R$ the reflectivity of the PC. The mode number can be estimated to be $5 \pm 1$ from the modal field pattern a in Fig. 3(d). From this, the reflectivity is calculated to be $0.8 \pm 0.1$.

Theoretically, for filling factors around 0.3 , as in our structures, the $\mathrm{H} 1$ defect cavity displays a single degenerate resonance in the center of the band gap. ${ }^{10,20}$ This dipole mode is known to have a relatively low $Q$-factor. ${ }^{22}$
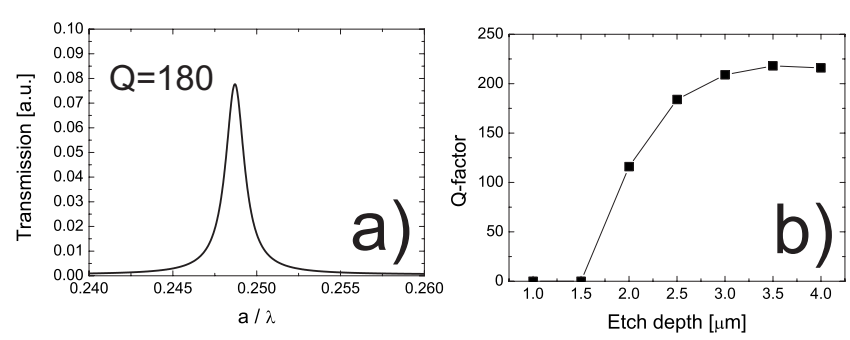

FIG. 6. (a) 3D-FDTD calculation of the $\mathrm{H} 1$ resonance. A $Q$-factor of 180 was obtained for 2.5- $\mu \mathrm{m}$-deep vertical holes, neglecting the tapered shape of the holes as displayed in Fig. 2(b). Splitting of the resonance peak due to discretization effects is artificially removed. (b) $Q$-factor of the $\mathrm{H} 1$ cavity vs the etch depth of the holes obtained by 3D-FDTD calculations.

H1 cavities were reported for InP membranes with $Q$-factors of 70 for an air filling factor of $f=0.43$ and $Q=100$ with $f=0.55 .^{10} Q$-factors of 270 and 250 for the $\mathrm{H} 1$ cavity were found for the AlGaAs/GaAs half-membrane ${ }^{11}$ and GaAs membrane ${ }^{13}$ systems respectively. From threedimensional (3D)-FDTD calculations for our experimental structure a $Q$-factor of 180 was obtained [see Fig. 6(a)] compared to a measured result of 65 . For this calculation the holes were approximated as cylinders with a depth of $2.5 \mu \mathrm{m}$. Since the hole shape of the actual structure suffers from tapering, ellipticity, and sidewall roughness the calculation is an upper limit of the $Q$-factor. Hole tapering is the main contribution to the deviation between calculation and experiment. $^{23}$

Given that improved etching for FP cavities has led to a dramatic increase in the $Q$-factor, ${ }^{6,7}$ it is likely that a similar improvement of the $Q$-factor may be possible for $\mathrm{H} 1$ cavities in these deeply etched systems. Berrier et al. ${ }^{24}$ show that an increase in the hole depth beyond $2.5 \mu \mathrm{m}$, which is obtained in this work, would lead to an increase in the $Q$-factor by approximately a factor of 2 . Variation in the hole depth in 3D-FDTD calculations [see Fig. 6(b)] show only a limited increase in the $Q$-factor beyond a depth of $2.5 \mu \mathrm{m}$ to a maximum of approximately 220. Thus the majority of improvements in $Q$-factor result from improved deep etching to a depth of $2.5 \mu \mathrm{m}$, approximately the vertical size of the modal field

Finally, the resonance frequency of the smallest possible cavity, i.e., the $\mathrm{HO}$ cavity, is shown as a function of $r^{\prime}$ in the right-most panel of Fig. 3(c). The radius $r^{\prime}$ and the shift in the holes $s^{\prime}$ are related as $r^{\prime}+s^{\prime}=r$, with $r$ the radius of the holes of the bulk PC [see Fig. 3(b)]. Figure 7 displays the spectrum of an $\mathrm{H} 0$ cavity, designed to have a value of $r^{\prime} / r=0.8$ and $s^{\prime} / r=0.2$; in the inset a SEM micrograph of the structure is given. From inspection of the SEM micrograph a value of approximately $r^{\prime} / r=0.7$ and $s^{\prime} / r=0.19$ was obtained.

As in Fig. 5, the spectrum is modulated by a beat and fine FP fringes stemming from the reflections at the PC and RWG facets. The $Q$-factor of the HO resonance is approximately 30, somewhat smaller than that found for the $\mathrm{H} 1$ cavity. The experimentally found resonance frequency deviates considerably from the value found by 2D-FDTD, which is attributed to deviations in the size and shape of the two shifted holes. Since the two holes with radius $r^{\prime}$ are much 


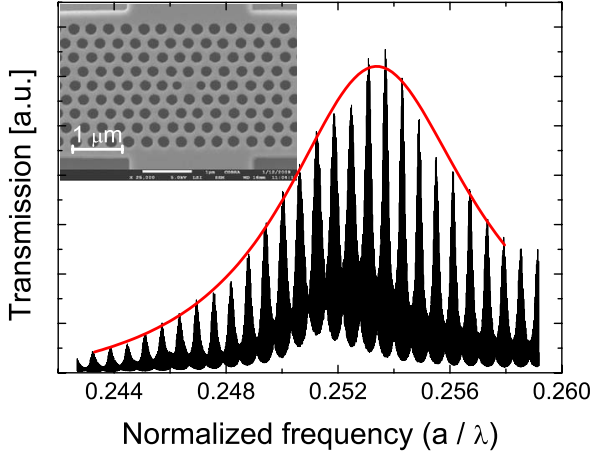

FIG. 7. (Color online) Spectrum of the HO cavity resonance. The $Q$-factor was determined from the envelope to be approximately 30 . This structure has a lattice constant of $380 \mathrm{~nm}$, bulk hole radius $r$ of $115 \mathrm{~nm}, r^{\prime} / r=0.7$, and $s^{\prime} / r=0.19$.

smaller than the bulk PC holes, the hole shape is expected to suffer from etching lag, which causes the holes to be less deep and more tapered. These two holes will present a much smaller average hole cross section to the electric field than can be estimated from SEM images from top. Due to the smaller hole diameter at the core layer, the effective cavity is larger, and the resulting resonance frequency will redshift.

In membrane-type $\mathrm{PCs}, \mathrm{H} 0$ cavities are reported to have a $Q$-factor that is at least an order of magnitude larger than unmodified $\mathrm{H} 1$ cavities. ${ }^{9,25}$ The large $Q$-factor of the $\mathrm{H} 0$ cavity in membrane devices is due to a gentle spatial decay of the modal electric field pattern. This gentle decay reduces the number of field components with a wave vector above the lightline, decreasing out-of-plane losses. ${ }^{26}$ In these low vertical index contrast systems this mode engineering does not apply, since the complete band gap is located above the lightline for the cladding.

In contrast to membranes, all three types of deeply etched cavities discussed here, have similar $Q$-factors, with a best result for the $\mathrm{H} 1$ cavity. The coupling of the cavities to the incoming WG mode is very different. For the FP1 cavity the coupling to the WG mode is large as it is in the path of all the light exciting the RWG. However, since the incoming mode is not a plane wave, there are large diffraction effects, while there is no confinement in the direction perpendicular to the RWGs which gives rise to leakage. For the $\mathrm{H} 1$ and $\mathrm{H} 0$ cavities the coupling to the WG mode is successively worse due to a smaller overlap between the modal field profile and the RWG mode. However, the confinement in the direction perpendicular to the RWGs is much better.

\section{B. Three row defects}

In this section the three row defects are systematically investigated, starting from a FP3 cavity, as shown in Fig. 1 (bottom row). This cavity is reproduced in Fig. 8(a) by the white holes. To obtain the H2 cavity, the FP3 cavity is modified by adding the colored holes except for the central hole. For each of the holes of a single color, the radii $r^{(4)}, r^{(3)}$, and $r^{(2)}$, are successively and gradually increased from 0 to $r$, the radius of the bulk crystal (white holes). Finally, the resonance frequency of the ring cavity is investigated by increasing the central hole with radius $r^{\prime}$. The impact of the addition

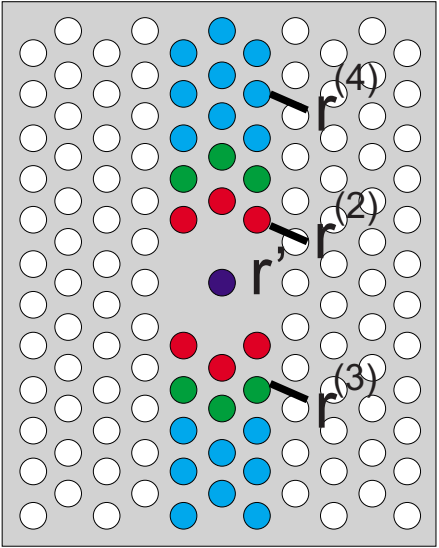

a)
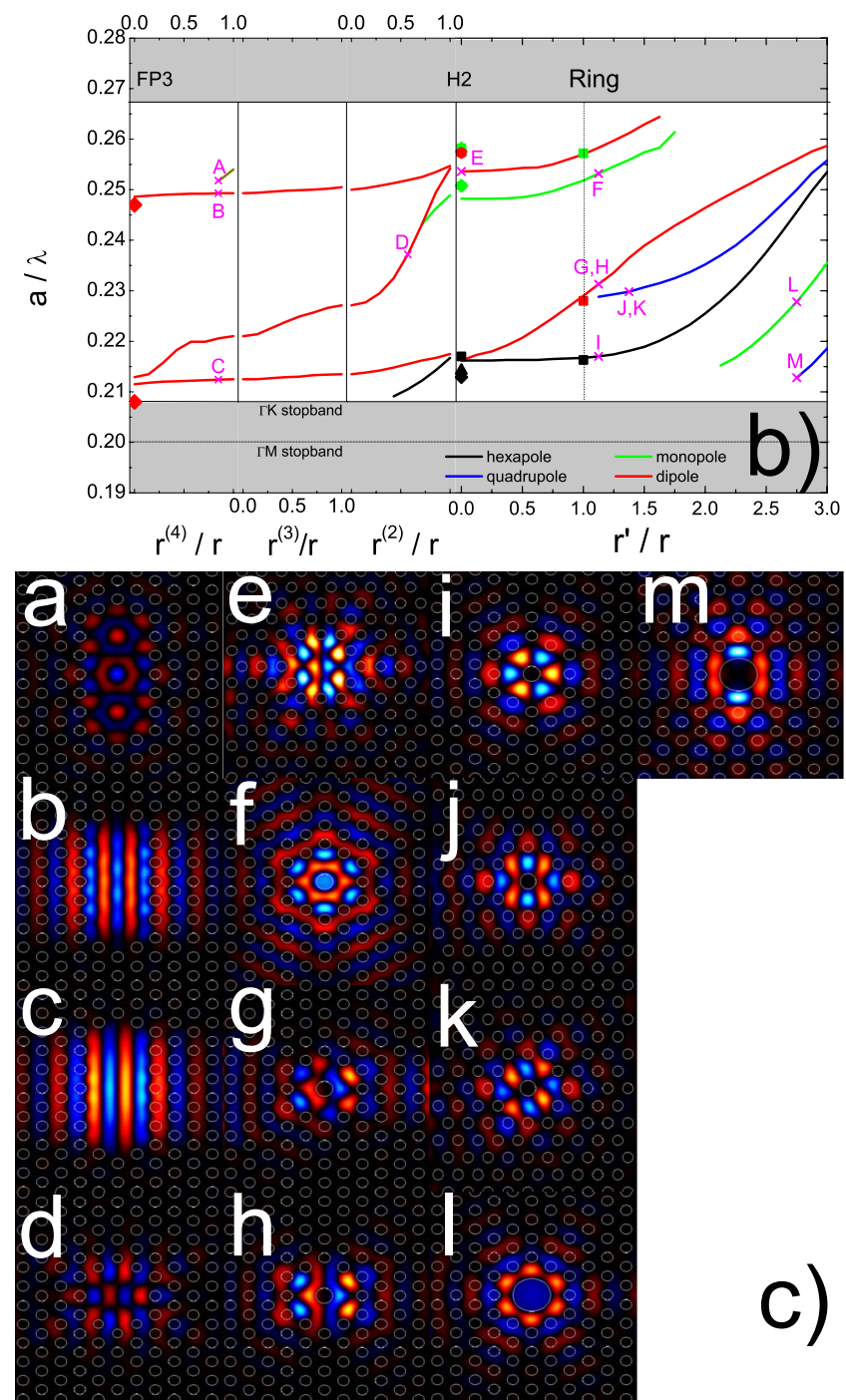

FIG. 8. (Color online) (a) Sketch of the three defect row cavities. By addition of the colored holes with radii $r^{(4)}, r^{(3)}$, and $r^{(2)}$ the FP3 cavity (white colored holes) is transformed into an $\mathrm{H} 2$ cavity. Finally, by addition of the center hole with radius $r^{\prime}$ the $\mathrm{H} 2$ cavity is changed into a ring cavity. (b) Evolution of the resonant modes from FP type cavity to $\mathrm{H} 2$ cavity to ring cavity as a function of the ratio of the radii of the holes, designated in a and the radius of the bulk holes $r$. The squares, triangles, and diamonds indicate the available experimental data, with each different symbol representing data from a different fabrication cycle. The gray areas denote the dielectric and airband. (c) Magnetic field maps $\left(H_{y}\right)$ of the various modes indicated by the crosses and capital letters in subfigure a. The mode patterns a-m correspond to $\mathrm{A}-\mathrm{M}$ indicated in (a). 


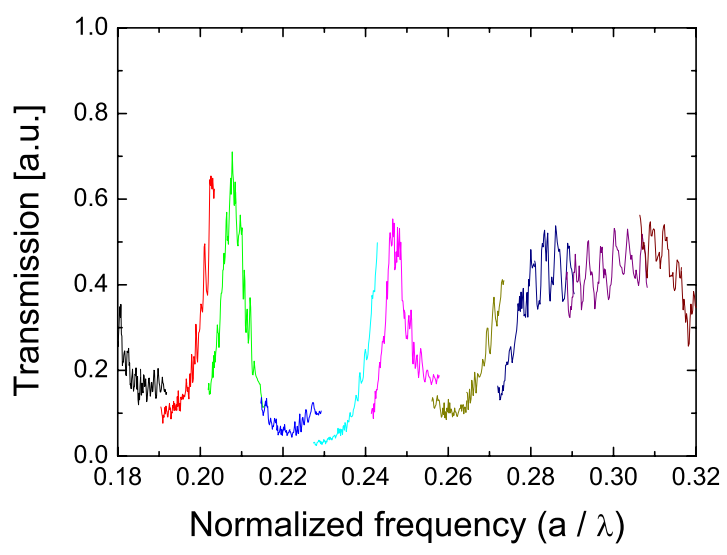

FIG. 9. (Color online) Wide band spectrum of a FP3 defect cavity. The differently colored parts of the spectrum result from structures with a varying lattice constant and radius (lithographic tuning), deviations from the hole diameter result in nonmatching parts of the spectrum.

of the holes and the gradual increase in the radius on the resonant frequency is analyzed by 2D-FDTD calculations.

Figure 8(b) shows the evolution of the modes of a simple three row defect FP-type cavity (FP3) toward hexagonal symmetric defect cavities, i.e., the $\mathrm{H} 2$ cavity and ring cavity successively. The lines connect the resonance frequencies found by 2D-FDTD calculations of the transmission of the PC and RWGs; each line consists of eight calculations per panel. The cavities transform into one another by modification of the radii of the holes indicated in Fig. 8(a). At selected positions indicated by the crosses and capital letters, the modal field pattern is displayed in Fig. 8(c). The modes a-m correspond to A-M in Fig. 8(b). The squares, triangles, and diamonds indicate the available experimental data of the FP3 cavity, the H2 cavity, and ring cavity. The different symbols indicate that the results were obtained from samples fabricated in independent production runs. The results from these measurements will be discussed in further detail below.

For the case of the FP3 cavity only modes with nodes in the $\Gamma K$ direction are expected. When viewed as a FP-type cavity with discrete mirrors, the mode number is determined by the cavity width $L$, i.e., $m=L n /(2 \lambda)$, with $n$ the refractive index and $\lambda$ the resonant wavelength. In the case of PC FP cavities, the penetration of the light into the PC mirrors also has to be taken into account. ${ }^{27}$ Figure 9 shows the spectrum of a FP3 cavity. For $a / \lambda<0.18$ and $a / \lambda>0.26$ the dielectric and air band edge respectively are visible. In the band gap two resonances with a $Q$-factor of approximately 30 are shown. The apparent discontinuities in the spectrum (peak and air band onsets) correspond to nonmatching parts of data from cavities with different lattice constant and are due to the usage of lithographic tuning technique to span the wide window of normalized frequency. In this fabricated device set, a $\mathrm{SiN}$ antireflection coating was applied to the end facets of the sample, removing the FP fringes resulting from reflections, as described previously.

As the ratio $r^{(4)} / r$ is increased, the FP-like mode is distorted, resulting in a small shift in the resonant frequencies. Additional modes ( $\mathrm{a}$ and $\mathrm{d}$ ), with nodes in the $\Gamma M$ direction, appear when $r^{(4)}$ is sufficiently large to have a stop band in the $\Gamma K$ direction. However, these modes are only weakly
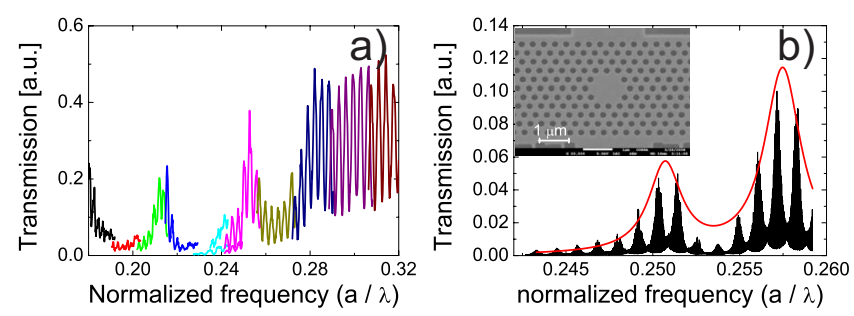

FIG. 10. (Color online) (a) Wide band spectrum of a H2 cavity. (b) Part of the spectrum of a $\mathrm{H} 2$ cavity (different sample) detailing the monopole and dipole resonances. The bold line is a Lorentzian fit of the envelope. In the inset an SEM image of the structure is shown.

excited due to the WG geometry, as their modal profile has limited overlap with the WG mode. Mode a in Fig. 8(c) shows a complicated modal pattern as a result of reflection in both directions reminiscent of monopole modes [compare with modes $\mathrm{f}$ and 1 in Fig. 8(c)]. Modes $\mathrm{b}$ and $\mathrm{c}$ display a slight perturbation of the FP-like modes of the FP3 cavity. The subsequent increase in the ratio $r^{(3)} / r$ shows a continuation of this trend. Confinement in the $\Gamma K$ direction is increased as shown by modes $\mathrm{d}$ and $\mathrm{e}$, which show dipole modes with nodes in the perpendicular direction.

With the increase in $r^{(2)} / r$ hexagonal symmetry is introduced to the cavity resulting in the appearance of a hexapole $(I)$ and monopole mode $(F)$ to the spectrum. For $r^{(2)} / r=1$, an $\mathrm{H} 2$ cavity is obtained [see inset of Fig. 10(b)]. The experimental spectrum of the $\mathrm{H} 2$ cavity is given in Fig. 10(a). In the band gap two resonance peaks with a $Q$-factor of approximately 30 are visible within the photonic band gap range from approximately $a / \lambda=0.20$ to $a / \lambda=0.28$. This graph reflects the spectrum of an $\mathrm{H} 2$ cavity with only three mirror rows (MR) resulting in this low $Q$-factor. The peak at $a / \lambda=0.21$ can be identified with the degenerate dipole/ hexapole mode in Fig. 8(b). Due to the modest $Q$-factor of the resonance at $a / \lambda=0.255$, the dipole and monopole mode are also degenerate. The designed $r / a$ value of 0.27 for this sample was slightly lower than used for the calculations, which accounts for the different resonance frequency. As with the FP3 spectrum in Fig. 9, an antireflection coating was applied on the cleaved facets of the access RWGs, however, a residual modulation is still present. The origin of this modulation is not relevant for the present work and is not further investigated, it is likely due to a modulation of the width of the RWGs.

Figure 10(b) shows the results obtained from a different sample, displaying the monopole and dipole mode resolved. To increase the $Q$-factor of the resonance, two extra rows of holes were added to the PC mirror with respect to Fig. 10(a), i.e., the cavity of Fig. 10 is terminated by three rows of holes while the cavity of Fig. 10(b) is terminated by four rows of holes, as seen in the inset. Since the $Q$-factor of these resonance peaks is substantially higher (approximately 100), the modes are now separated.

The measured $Q$-factor of the $\mathrm{H} 2$ cavity in this deeply etched system is somewhat smaller than values found for InP membranes on Si (300 to 400). ${ }^{10}$ Higher values are found for the AlGaAs/GaAs half-membrane system ${ }^{11}$ and InP air 

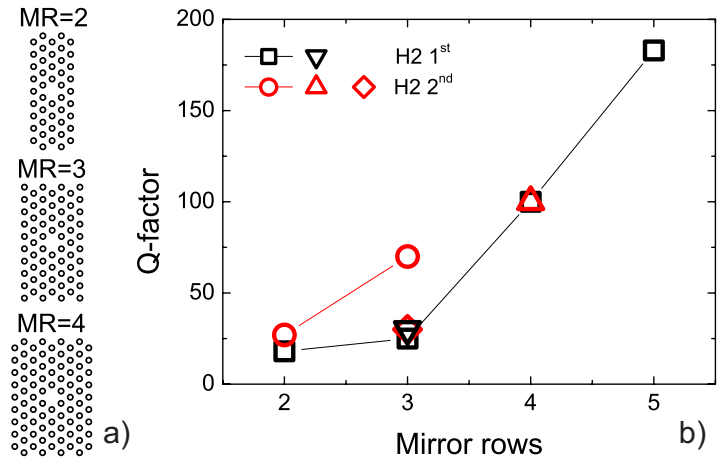

FIG. 11. (Color online) (a) Sketch of the variation in MR. (b) $Q$-factor of resonance peaks in the spectrum of $\mathrm{H} 2$ defect cavities vs the MR on either side of the defect. The squares and reversed triangles denote the $Q$-factors of the first order (dipole/hexapole) resonance and the circles, triangles, and diamonds display the second order (merged dipole and monopole) resonances of the $\mathrm{H} 2$ cavity for several samples.

membranes, ${ }^{12}$ in which a $Q$-factor of approximately 600 for the $\mathrm{H} 2$ cavity was found. Even larger $Q$-factors, up to $4000,{ }^{13}$ are found in GaAs membranes.

The $Q$-factor of the $\mathrm{H} 2$-cavity resonances was investigated as a function of the width of the PC mirrors. The number of rows on either side of the defect (MR) is varied from 2 to 5 [see sketch in Fig. 11(a)]. The squares and reversed triangles in Fig. 11(b) denote the $Q$-factors of the first order (dipole/hexapole) resonance and the circles, triangles, and diamonds display the second order (merged dipole and monopole) resonances of the $\mathrm{H} 2$ cavity for several samples. Both data sets show approximately the same expected behavior, namely a rise of the $Q$-factor as a result of better light confinement in the defect due to increased mirror reflectivity. For the merged dipole and monopole $\mathrm{H} 2$ resonances (second order) the data set is incomplete as for larger mirror thickness, the resonance peak is difficult to distinguish in the spectrum. This can be attributed to reduced coupling efficiency between the access WGs and the defect cavity and losses. High in-plane losses are incurred, since the second order feature is spectrally located close to the airband, which may induce leakage. The data in Fig. 11(b) show a monotonic increase in the $Q$-factor with increasing number of MR, while saturation is not evident.

From both calculations and measurements done for $\mathrm{H} 2$ cavities in membrane PCs measured by photoluminescence (PL), more modes than presented in Fig. 8(b) were reported. ${ }^{12}$ However, only a subset of modes were found, of which some modes are not resolved due to the low $Q$-factor. Opposed to PL, the excitation of the cavity using WGs favors modes with a strong coupling to the modal field of the WG.

The FP fringes as seen in Fig. 10(b) can also be used to estimate the reflectivity of the PC, by analysis of the maximum to minimum ratio of the fine fringes obtained from a zoom in of Fig. 10(a), prior to application of the antireflection coating (see Fig. 12). This figure closely resembles the inset of Fig. 5, if plotted on a linear scale. From this figure the largest minimum to maximum ratio of the fringes was found to be approximately 80 .

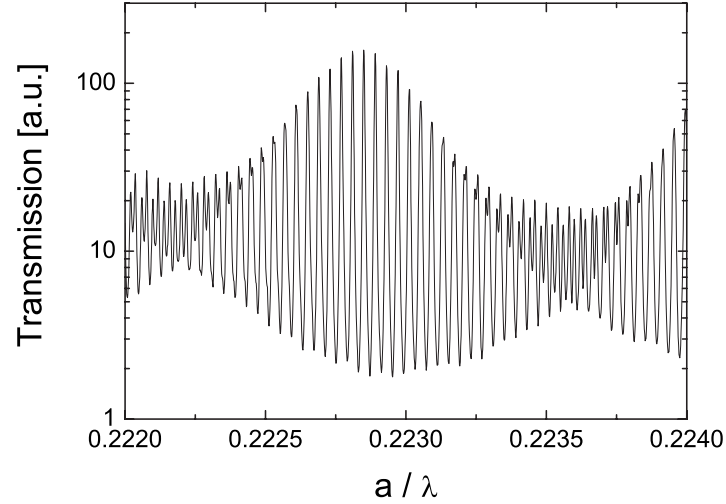

FIG. 12. Typical FP fringe pattern present in the measurements of FP3 and $\mathrm{H} 2$ cavity spectra; note that the transmission is plotted on a logarithmic scale. The largest minimum to maximum ratio for these fringes is approximately 80 .

In order to explain this large ratio, we model the access WGs as cavities of lengths $L_{1}$ and $L_{2}$ [RWG1 and RWG2 in Fig. 2(a)], with the end facets and PC as planar mirrors with a reflection coefficient of $R_{f}$ and $R_{\mathrm{PC}}$, respectively. The system of these two coupled cavities constitute a compound cavity. The transmission $T$ through one such a cavity of length $L$ is given by the FP formula ${ }^{28}$

$$
T=\frac{\left(1-R_{f}\right)\left(1-R_{\mathrm{PC}}\right) e^{-\alpha L}}{1-2 \sqrt{R_{f} R_{\mathrm{PC}}} e^{-\alpha L} \cos \left(\frac{4 \pi L n_{g}}{\lambda}\right)+R_{f} R_{\mathrm{PC}} e^{-2 \alpha L}}
$$

with $\alpha$ the coefficient of the WG losses and $n_{g}$ the group index. In the limit of weak coupling, i.e., $R_{\mathrm{PC}} \sim 1$, a simple model of the coupled cavity is to use the output of the first cavity as input for the second. In this approach the simple FP formula with $L=L_{1}$ is multiplied with the identical formula with $L=L_{2}$. This turns out to be a very accurate description of the FP fringes. The result was verified employing a scattering matrix calculation, using the theory of multilayer films. ${ }^{28}$ The propagation losses in the WGs were estimated from examination of the fringe pattern of a single RWG to be lower than $2 \mathrm{~dB} / \mathrm{cm}$ and are considered negligible for the current analysis. The reflection coefficient of the end facets $R_{f}$ is 0.33 and was calculated taking into account modal reflection and WG dispersion. The cavity lengths $L_{1}$ and $L_{2}$ were measured to be $1.135 \pm 0.005$ and $1.165 \pm 0.005 \mathrm{~mm}$, respectively. The reflectivity of the PC, $R_{\mathrm{PC}}$, and the group index, $n_{g}$ can now be simultaneously determined by fitting the measured fringes. For the group index, $n_{g}=3.674 \pm 0.001$ was found and the reflectivity was found to be $0.72 \pm 0.05$.

Alternatively, the reflectivity of the PC mirrors can be found directly from the measured spectrum by determination of the finesse, $F$, as both the FP3 cavity (Fig. 9) and the H2 cavity [Fig. 10(a)] show two peaks of consecutive order. Since two resonance orders are available, it is not necessary to estimate the mode number $m$, as in Eq. (1). The reflectivity is calculated by 


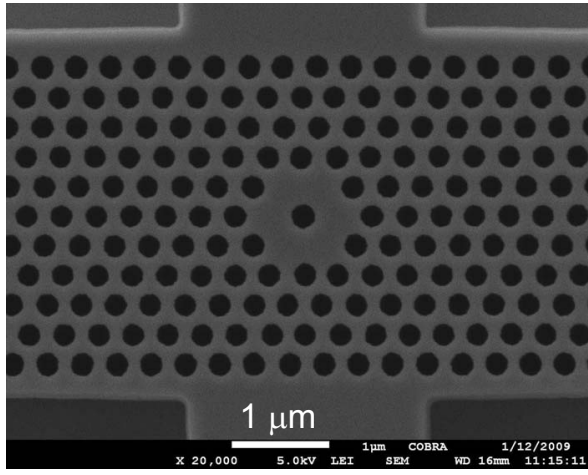

FIG. 13. SEM micrograph of the ringlike cavity. The scale of the image is indicated by the white bar in the black caption.

$$
F=\pi \frac{\sqrt{R}}{1-R}
$$

The reflection of the FP3 and H2 structures is found to be $0.70 \pm 0.05$, consistent with the value found from the analysis of the fringes. These values are comparable to reflectivity coefficients found by other work with non-optimized etching technology $(R=0.63 \pm 0.02){ }^{2}$ However, the reflectivity $(R=0.91)$ is not as high as might be expected from comparison with Ref. 29.

Finally, the addition of the single hole $\left(r^{\prime}\right)$ in the center of the $\mathrm{H} 2$ cavity forms a ringlike cavity [see Fig. 8(b)]. Since the effective refractive index of the cavity is lowered by increasing the ratio $r^{\prime} / r$, the modes blueshift toward the air band. ${ }^{30-32}$ For $r^{\prime} / r=1$, a ring defect cavity consisting of bulk holes is obtained (see Fig. 13).

Figure 14 gives the spectrum of this $\mathrm{PC}$ ring resonator with an inner radius and ring width of the order of the wavelength. This type of cavity supports four resonant frequencies in the band gap. In the insets, the modal patterns of the resonances are depicted. These modes can be identified as a hexapole, dipole, and a degenerate monopole/dipole respectively. The $Q$-factor of the resonances were obtained by fitting the envelope with a Lorentzian, yielding 300, 75, and 30 from left to right, respectively.

In the work reported here, we have detected three resonances in the band gap due to relatively high optical losses, leading to a decrease in $Q$-factor with increasing frequency. For the InP/InGaAsP/InP system the out-of-plane losses are

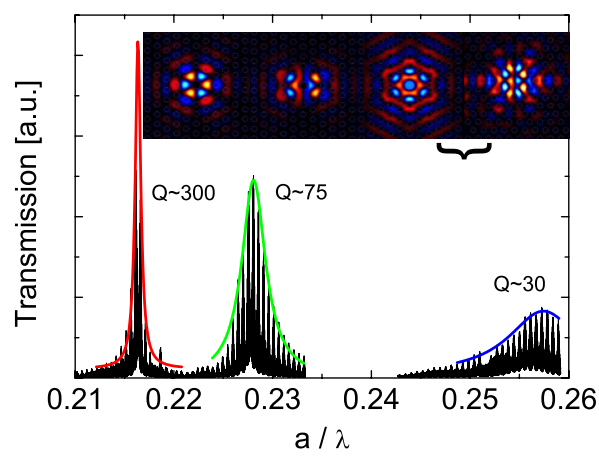

FIG. 14. (Color online) Spectrum of the ring cavity. The $Q$-factor was determined from the envelope to be approximately 300, 75, and 30 from left to right. The insets displays the modal patterns of the resonances. inherently large compared to membranes. For perfect PC holes, it is expected that modes far away from the band edges have the optimal confinement and thus highest $Q$-factor. However, fabrication imperfections (tapered holes, ellipticity, and surface roughness) have a significant impact on the losses for modes that concentrate their modal fields near or in the PC holes, i.e., modes near the air band. Therefore modes closest to the dielectric band edge are expected to yield higher $Q$-factors. A moderate $Q$-factor of 160 is sufficient to enable the operation of high efficiency all-optical logic gates, ${ }^{3}$ which makes this cavity type suitable for applications such as gating logic. In comparison, for $\mathrm{Si}$ membranes $Q$-factors of 400 and 2000 were found in combination with infiltration of the enlarged central hole. ${ }^{14,15}$

Figure 8(a) shows that a further increase in the radius of the center hole leads to a strong blueshift of the modes of the ring cavity. Also, several modes successively appear into the band gap: modes $\mathrm{J}$ and $\mathrm{K}$ with a quadrupole symmetry, monopolar mode L, and quadrupolar mode $\mathrm{M}$. The spectral location of all modes show a large dependence on the radius of the enlarged central hole. This makes these modes very suitable for sensor applications, since changes in the effective refractive index are proportional to $\Delta r^{\prime} / r$. Thus the introduction of a substance with a higher different refractive into the central hole will have a large impact on these modes. ${ }^{15}$

\section{CONCLUSIONS}

In conclusion, we have explored the cavity resonances of wavelength-sized cavities in the deeply etched InP system and analyzed their similarities. Two classes of cavities, with a radius of one and two unit cells in the transmission direction, were fabricated and studied by transmissions spectroscopy and analyzed in combination with FDTD calculations. The single unit cell defect class includes the FP1, H1, and $\mathrm{H} 0$ cavities. The class of defect cavities of the size of two unit cells includes the FP3, H2, and ring cavities.

For the single unit cell defect class, H0, H1, and FP1 cavities have similar $Q$-factors, about 50 . The best result with $Q \sim 65$ was obtained for the H1 cavity, which is comparable to results for membranes.

For the defect class of the size of two unit cells, the $Q$-factors of the FP3, H2, and ring cavity vary by one order of magnitude and mainly due to differences in sample quality. The best result with $Q \sim 300$ was obtained for the ring cavity.

\section{ACKNOWLEDGMENTS}

The authors would like to thank P. Nouwens and T. de Vries for contributions to the experimental work. This work is part of the research program of the "Stichting voor Fundamenteel Onderzoek der Materie (FOM)." which is financially supported by the "Nederlandse Organisatie voor Wetenschappelijk Onderzoek (NWO)." Part of this research is supported by NanoNed, a technology program of the Dutch Ministry of Economic Affairs via the foundation STW.

${ }^{1}$ J. D. Joannopoulos, S. G. Johnson, R. D. Meade, and J. N. Winn, Photonic Crystals: Molding the Flow of Light (Princeton University Press, Princeton, 2008). 
${ }^{2}$ R. Ferrini, D. Leuenberger, M. Mulot, M. Qiu, J. Moosburger, M. Kamp, A. Forchel, S. Anand, and R. Houdré, IEEE J. Quantum Electron. 38, 786 (2002).

${ }^{3}$ Z. Qiang, W. Zhou, and R. A. Soref, Opt. Express 15, 1823 (2007).

${ }^{4}$ A. Ghaffari, F. Monifi, M. Djavid, and M. S. Abrishamian, Opt. Commun. 281, 5929 (2008).

${ }^{5}$ P. Andalib and N. Granpayeh, J. Opt. Soc. Am. B 26, 10 (2009).

${ }^{6}$ J. Martz, R. Ferrini, F. Nüesch, L. Zuppiroli, B. Wild, L. A. Dunbar, R. Houdré, M. Mulot, and S. Anand, J. Appl. Phys. 99, 103105 (2006).

${ }^{7}$ A. Berrier, M. Mulot, S. Anand, A. Talneau, R. Ferrini, and R. Houdré, J. Vac. Sci. Technol. B 25, 1 (2007).

${ }^{8}$ M. Mulot, M. Qiu, M. Swillo, B. Jaskorzynska, S. Anand, and A. Talneau, Appl. Phys. Lett. 83, 1095 (2003).

${ }^{9}$ K. Nozaki, S. Kita, and T. Baba, Opt. Express 15, 7506 (2007).

${ }^{10}$ C. Monat, C. Seassal, X. Letrarte, P. Regreny, M. Gendry, P. Rojo Romeo, P. Viktorovitch, M. Le Vassor d'Yerville, D. Cassagne, J. P. Albert, E. Jalaguier, S. Pocas, and B. Aspar, J. Appl. Phys. 93, 23 (2003).

${ }^{11}$ A. Kress, F. Hofbauer, N. Reinelt, H. J. Krenner, M. Bichler, D. Schuh, R. Meyer, G. Abstreiter, and J. J. Finley, Physica E (Amsterdam) 26, 351 (2005).

${ }^{12}$ C. Grillet, P. Pottier, X. Letartre, C. Seassal, P. Rojo-Romeo, P. Viktorovitch, M. Le Vassor d'Yerville, and D. Cassagne, Eur. Phys. J.: Appl. Phys. 16, 37 (2001)

${ }^{13}$ C. Reese, B. Gayral, B. D. Gerardot, A. Imamoğlu, P. M. Petroff, and E. Hu, J. Vac. Sci. Technol. B 19, 2749 (2001).

${ }^{14}$ S. Vignolini, F. Riboli, F. Intonti, M. Belotti, M. Gurioli, Y. Chen, M Colocci, L.C. Andreani, and D.S. Wiersma, Phys. Rev. E 78, 045603(R) (2008).

${ }^{15}$ M. R. Lee and P. M. Fauchet, Opt. Lett. 32, 3284 (2007).

${ }^{16}$ K. Inoshita and T. Baba, IEEE J. Quantum Electron. 9, 1347 (2003).

${ }^{17}$ H. H. J. E. Kicken, I. Barbu, R. W. van der Heijden, F. Karouta, R. Nötzel, E. W. J. M. van der Drift, and H. W. M. Salemink, Opt. Lett. 34, 2207 (2009).
${ }^{18}$ C. F. Carlström, R. van der Heijden, F. Karouta, R. W. van der Heijden, and H. W. M. Salemink, and E. van der Drift, J. Vac. Sci. Technol. B 24, L6 (2006).

${ }^{19}$ M. Qiu, Appl. Phys. Lett. 81, 1163 (2002).

${ }^{20}$ O. Painter, J. Vuckovic, and A. Scherer, J. Opt. Soc. Am. B 16, 275 (1999).

${ }^{21}$ A. Talneau, M. Mulot, S. Anand, and Ph. Lalanne, Appl. Phys. Lett. 82, 2577 (2003).

${ }^{22}$ W. H. Zheng, G. Ren, X. T. Ma, X. H. Cai, L. H. Chen, K. Nozaki, and T. Baba, J. Cryst. Growth 292, 341 (2006)

${ }^{23}$ R. Ferrini, R. Houdré, H. Benisty, M. Qiu, and J. Moosburger, J. Opt. Soc. Am. B 20, 469 (2003).

${ }^{24}$ A. Berrier, R. Ferrini, A. Talneau, R. Houdré, and S. Anand, J. Appl. Phys. 103, 096106 (2008)

${ }^{25}$ Z. Zhang and M. Qiu, Opt. Express 12, 3988 (2004).

${ }^{26}$ Y. Akahane, T. Asano, B.-S. Song, and S. Noda, Nature (London) 425, 944 (2003).

${ }^{27}$ M. Rattier, H. Benisty, C. J. M. Smith, A. Béraud, D. Cassagne, C. Jouanin, T. F. Krauss, and C. Weisbuch, IEEE J. Quantum Electron. 37, 237 (2001).

${ }^{28}$ L. M. Pedrotti, L. S. Pedrotti, and F. L. Pedrotti, Introduction to Optics (Pearson Education Ltd., Harlow, 2006).

${ }^{29}$ R. Ferrini, B. Lombardet, B. Wild, R. Houdré, S. Olivier, H. Benisty, A. Djoudi, L. Legouézigou, S. Hubert, S. Sainson, J.-P. Chandouineau, S. Fabre, F. Pommereau, and G.-H. Duan, Electron. Lett. 38, 962 (2002).

${ }^{30}$ S.-H. Kim, H.-Y. Ryu, H.-G. Park, G.-H. Kim, T.-S. Choi, Y.-H. Lee, and J.-S. Kim, Appl. Phys. Lett. 81, 2499 (2002).

${ }^{31}$ A. R. Alija, L. J. Martinez, P. A. Postigo, C. Seassal, and P. Viktorovitch, Appl. Phys. Lett. 89, 101102 (2006).

${ }^{32}$ W.-Y. Chiu, T.-W. Huang, Y.-H. Wu, Y.-J. Chan, C.-H. Hou, H.-T. Chien, and C.-C. Chen, Opt. Express 15, 15500 (2007). 